\title{
A Dutch book coherence condition for conditional completely alternating Choquet expectations
}

\author{
G. Coletti ${ }^{1}$ (D) D. Petturiti ${ }^{2}$ (D) B. Vantaggi $^{3}$
}

Received: 28 April 2020 / Accepted: 21 July 2020 / Published online: 5 August 2020

(c) The Author(s) 2020

\begin{abstract}
Stemming from de Finetti's coherence for finitely additive (conditional) probabilities, the paradigm of coherence has been extended to other uncertainty calculi. We study the notion of coherence for conditional completely alternating Choquet expectations, providing an avoiding Dutch book like condition.
\end{abstract}

Keywords Coherence $\cdot$ Completely alternating Choquet expectations · Conditioning · Dutch book

Mathematics Subject Classification 60A05 · 28E10 - 90C05

\section{Introduction}

In the second half of the past century, decision theory and artificial intelligence saw the introduction of different non-additive measures and integrals, generalizing probability and expectation. That was motivated by the need to manage knowledge acquisition and decision processes when the available information is incomplete, imprecise or expressed in vague terms. In this context, the Dempster-Shafer theory of evidence $[7,17,18]$ is the most known framework, relying on two non-additive dual set functions $\mathrm{Bel}$ and $\mathrm{Pl}$, said belief and plausibility functions. Belief and plausibility functions are connected to finitely additive (f.a. for short) probabilities, since every f.a. probability $P$ on an algebra $\mathcal{A}$ determines a belief and a

To the memory of Domenico "Mimmo" Candeloro, an estimated colleague and a dear friend.

$凶$ B. Vantaggi

barbara.vantaggi@uniroma1.it

G. Coletti

giulianella.coletti@unipg.it

D. Petturiti

davide.petturiti@unipg.it

1 Department of Mathematics and Computer Science, University of Perugia, Perugia, Italy

2 Department of Economics, University of Perugia, Perugia, Italy

3 Department of MEMOTEF, "La Sapienza” University of Rome, Rome, Italy 
plausibility function on every super-algebra $\mathcal{B}$, with $\mathcal{A} \subset \mathcal{B} \subseteq \mathcal{P}(\Omega)$, through its inner and outer measures. Moreover, every belief function $\mathrm{Bel}$ (or, equivalently, its dual plausibility $P l$ ) on an algebra $\mathcal{A}$ induces a closed (in the product topology) convex set of finitely additive probability measures on $\mathcal{A}$ said core:

$$
\operatorname{core}(B e l)=\{P: P \text { is a f.a. probability on } \mathcal{A}, B e l \leq P \leq P l\},
$$

and it holds that $B e l=\min \operatorname{core}(B e l)$ and $P l=\max \operatorname{core}(B e l)$, where the minimum and maximum are pointwise on the elements of $\mathcal{A}$ [16]. Starting from $\mathrm{Bel}$ and $\mathrm{Pl}$, different functional extensions to the set of bounded real-valued functions (said gambles) are possible, through suitable notions of integral (see, e.g., [12]). Here, we refer to the Choquet integral, for its lower/upper expectation interpretation

$$
\oint X \mathrm{~d} B e l=\min _{P \in \operatorname{core}(B e l)} \int_{\Omega} X \mathrm{~d} P \text { and } \oint X \mathrm{~d} P l=\max _{P \in \operatorname{core}(B e l)} \int_{\Omega} X \mathrm{~d} P,
$$

and for its well-recognized role in decision-theoretic and economic applications (see, e.g.,[2, $4,10,12])$. Belief and plausibility functions miss a commonly accepted notion of conditioning and the debate on the "best" choice is still open (for a discussion on the different axiomatic definitions see [3]). In this article we adopt a notion of conditioning that allows us both to make inference using a Bayes like procedure, and to reason under hypotheses, taking into account also unexpected events (i.e., events with null plausibility).

The aim of this paper is to deal with partial, imprecise knowledge, taking into account conditioning. The root of such problems has been addressed in the probabilistic context by de Finetti [6]. He introduced the concept of coherence which characterizes, in terms of absence of Dutch books, the consistency with a (conditional) expectation, of an assessment defined only on the (conditional) gambles on which one has information and interest. Following this approach, we introduce a notion of coherence expressed in terms of a generalized betting scheme, and we prove that avoiding generalized Dutch books characterizes partial assessments consistent with a conditional completely alternating Choquet expectation.

The paper is structured as follows. Section 2 collects the necessary preliminaries, while Sect. 3 introduces conditional completely alternating Choquet expectations. Finally, Sect. 4 deals with coherence and its characterization in terms of a generalized form of Dutch book.

\section{Preliminaries}

Let $\Omega$ be an arbitrary non-empty set. In what follows, $\mathcal{P}(\Omega)$ stands for the power set of $\Omega$ and, for every algebra $\mathcal{A} \subseteq \mathcal{P}(\Omega)$, let $\mathcal{A}^{0}=\mathcal{A} \backslash\{\emptyset\}$. Moreover, for every $A \in \mathcal{A}$, we denote by $\mathbf{1}_{A}: \Omega \rightarrow\{0,1\}$ its indicator.

A plausibility function is a mapping $P l: \mathcal{A} \rightarrow[0,1]$ such that:

(i) $\operatorname{Pl}(\emptyset)=0$ and $\operatorname{Pl}(\Omega)=1$;

(ii) for every $k \geq 2$ and for every $A_{1}, \ldots, A_{k} \in \mathcal{A}$,

$$
P l\left(\bigcap_{i=1}^{k} A_{i}\right) \leq \sum_{\emptyset \neq I \subseteq\{1, \ldots, k\}}(-1)^{|I|+1} P l\left(\bigcup_{i \in I} A_{i}\right) .
$$

Condition (ii) above is usually termed complete alternancy. The dual of a plausibility function Bel $: \mathcal{A} \rightarrow[0,1]$ defined, for every $A \in \mathcal{A}$, as $\operatorname{Bel}(A)=1-\operatorname{Pl}\left(A^{c}\right)$, is called belieffunction and is completely monotone, i.e., it satisfies a property analogous to (ii), obtained by inverting the inequality and by switching intersections and unions. 
Belief and plausibility functions have been introduced in $[7,17,18]$. We stress that, in this paper no form of continuity is required for $\mathrm{Bel}$ and $\mathrm{Pl}$.

Every plausibility function $\mathrm{Pl}$ and its dual belief function $\mathrm{Bel}$ on $\mathcal{A}$ are (see Theorem A in [11]) in bijection with a finitely additive probability measure $\mu$ defined on an algebra $\mathfrak{A}$ possibly strictly contained in $\mathcal{P}(\mathcal{U})$, where $\mathcal{U}=\mathcal{A}^{0}$. The algebra $\mathfrak{A}$ is built as follows: for every $A \in \mathcal{A}$, define $A^{\mathbf{L}}, A^{\mathbf{U}} \in \mathcal{P}(\mathcal{U})$, as

$$
A^{\mathbf{L}}=\{B \in \mathcal{U}: B \subseteq A\} \text { and } A^{\mathbf{U}}=\{B \in \mathcal{U}: B \cap A \neq \emptyset\}
$$

and let $\mathfrak{A}$ be the algebra generated by $\left\{A^{\mathbf{L}}: A \in \mathcal{U}\right\}$ (or, equivalently, by $\left\{A^{\mathbf{U}}: A \in \mathcal{U}\right\}$ ). The finitely additive probability $\mu$ on $\mathfrak{A}$ allows to provide an integral expression of $\mathrm{Bel}$ and $P l$ obtained, for every $A \in \mathcal{A}$, as

$$
\operatorname{Bel}(A)=\int_{\mathcal{U}}\left(\inf _{\omega \in B} \mathbf{1}_{A}(\omega)\right) \mu(\mathrm{d} B) \text { and } P l(A)=\int_{\mathcal{U}}\left(\sup _{\omega \in B} \mathbf{1}_{A}(\omega)\right) \mu(\mathrm{d} B),
$$

where the integrals are of Stieltjes type [1]. The finitely additive probability $\mu$ on $\mathfrak{A}$ is called the Möbius inverse of $\mathrm{Bel}$. This paper adopts the conditioning rule expressed by the axiomatic definition of conditional plausibility or belief functions given in [5], which generalizes the Dempster rule [7], allowing for conditioning to events of null plausibility.

Definition 2.1 Let $\mathcal{A} \subseteq \mathcal{P}(\Omega)$ be an algebra and $\mathcal{H} \subseteq \mathcal{A}^{0}$ be an additive class (i.e., a set of events closed under finite unions). A function $P l: \mathcal{A} \times \mathcal{H} \rightarrow[0,1]$ is a conditional plausibility function if it satisfies the following conditions:

(i) $P l(E \mid H)=P l(E \cap H \mid H)$, for every $E \in \mathcal{A}$ and $H \in \mathcal{H}$;

(ii) $P l(\cdot \mid H)$ is a plausibility function on $\mathcal{A}$, for every $H \in \mathcal{H}$;

(iii) $P l(E \cap F \mid H)=P l(E \mid H) \cdot P l(F \mid E \cap H)$, for every $E \cap H, H \in \mathcal{H}$ and $E, F \in \mathcal{A}$.

Given a conditional plausibility function, the dual conditional belief function $\operatorname{Bel}(\cdot \mid \cdot)$ is defined, for every $E \mid H \in \mathcal{A} \times \mathcal{H}$, as $\operatorname{Bel}(E \mid H)=1-P l\left(E^{c} \mid H\right)$.

In case the additive class $\mathcal{H}$ is finite, then a conditional plausibility $P l(\cdot \mid \cdot)$ is completely characterized by a linearly ordered class $\left\{P l_{0}, \ldots, P l_{k}\right\}$ of plausibility functions on $\mathcal{A}$, said minimal agreeing class [5]. Given $P l(\cdot \mid \cdot)$ set:

- $P l_{0}(\cdot)=P l\left(\cdot \mid H_{0}^{0}\right)$ with $H_{0}^{0}=\bigcup_{H \in \mathcal{H}} H$;

- $P l_{\alpha}(\cdot)=P l\left(\cdot \mid H_{0}^{\alpha}\right)$, for $\alpha>0$, where $H_{0}^{\alpha}=\bigcup\left\{H \in \mathcal{H}: P l_{\beta}(H)=0, \beta=\right.$ $0, \ldots, \alpha-1\} \neq \emptyset$, and the construction stops at index $k$ such that $H_{0}^{k+1}=\varnothing$.

Such a class satisfies $P l_{\alpha}\left(H_{0}^{\alpha}\right)=1$ and $P l_{\alpha}\left(\left(H_{0}^{\alpha}\right)^{c}\right)=0$. Vice versa, starting from a minimal agreeing class $\left\{P l_{0}, \ldots, P l_{k}\right\}$, for every $E \mid H \in \mathcal{A} \times \mathcal{H}$, denoting by $\alpha_{H}$ the minimum index in $\{0, \ldots, k\}$ such that $P l_{\alpha_{H}}(H)>0$, it holds that

$$
P l(E \mid H)=\frac{P l_{\alpha_{H}}(E \cap H)}{P l_{\alpha_{H}}(H)} .
$$

This shows, in case of a finite $\mathcal{H}$, the presence of a one-to-one correspondence between the class of conditional plausibility functions on $\mathcal{A} \times \mathcal{H}$ and the class of minimal agreeing classes on $\mathcal{A}$ (see also $[5,15])$.

\section{Completely alternating Choquet expectations}

A gamble $X: \Omega \rightarrow \mathbb{R}$ is a state-contingent (possibly positive or negative) payoff expressed in a linear utility scale. The operations between gambles, such as sum, multiplication, and 
multiplication by a constant, as well as ordering comparisons, are always assumed pointwise on $\Omega$. Let $\mathcal{L}(\Omega)$ be the set of all bounded gambles which is a topological linear space under the topology of uniform convergence induced by the supremum norm $\|X\|=\sup _{\omega \in \Omega}|X(\omega)|$. In particular, denote by $\mathcal{L}(\Omega)_{+}=\{X \in \mathcal{L}(\Omega): X \geq 0\}$ the convex cone of non-negative bounded gambles. We further denote by $\mathcal{L}(\Omega)^{*}$ the topological dual space of $\mathcal{L}(\Omega)$. If we set $\mathcal{U}=\mathcal{P}(\Omega)^{0}$, then $\mathcal{L}(\mathcal{U}), \mathcal{L}(\mathcal{U})_{+}$and $\mathcal{L}(\mathcal{U})^{*}$ have analogous meaning. Let Bel and $P l$ be a pair of dual belief and plausibility functions on $\mathcal{P}(\Omega)$, then denote by $\mathbf{C}_{B e l}$ and $\mathbf{C}_{P l}$ the functionals on $\mathcal{L}(\Omega)$, determined by the Choquet integral with respect to $\mathrm{Bel}$ and $\mathrm{Pl}$, respectively: for every $X \in \mathcal{L}(\Omega)$,

$$
\mathbf{C}_{B e l}(X)=\oint X \mathrm{~d} B e l \text { and } \mathbf{C}_{P l}(X)=\oint X \mathrm{~d} P l .
$$

For every $k \geq 2$, for every $X_{1}, \ldots, X_{k} \in \mathcal{L}(\Omega)$, it holds that

$$
\mathbf{C}_{P l}\left(\bigwedge_{i=1}^{k} X_{i}\right) \leq \sum_{\emptyset \neq I \subseteq\{1, \ldots, k\}}(-1)^{|I|+1} \mathbf{C}_{P l}\left(\bigvee_{i \in I} X_{i}\right)
$$

where $\wedge$ and $\vee$ denote the pointwise minimum and maximum of functions. $\mathbf{C}_{B e l}$ satisfies an analogous property, obtained by inverting the inequality and by switching minima and maxima. Then, we refer to $\mathbf{C}_{B e l}$ and $\mathbf{C}_{P l}$ as completely monotone and completely alternating Choquet expectations. Notice that the two functionals are dual, that is, for every $X \in \mathcal{L}(\Omega)$, it holds $\mathbf{C}_{B e l}(X)=-\mathbf{C}_{P l}(-X)$. Moreover, they can be interpreted as lower and upper expectation functionals, since, for every $X \in \mathcal{L}(\Omega)$, it holds

$$
\mathbf{C}_{B e l}(X)=\min _{P \in \operatorname{core}(B e l)} \int_{\Omega} X \mathrm{~d} P \text { and } \quad \mathbf{C}_{P l}(X)=\max _{P \in \operatorname{core}(B e l)} \int_{\Omega} X \mathrm{~d} P .
$$

For $X \in \mathcal{L}(\Omega)$, the corresponding lower and upper generalized gambles $X^{\mathbf{L}}, X^{\mathbf{U}} \in \mathcal{L}(\mathcal{U})$ are defined, for every $B \in \mathcal{U}$, as

$$
X^{\mathbf{L}}(B)=\inf _{\omega \in B} X(\omega) \text { and } X^{\mathbf{U}}(B)=\sup _{\omega \in B} X(\omega) .
$$

If $\mu$ is the Möbius inverse of $\mathrm{Bel}$ defined on the algebra $\mathfrak{A}$ generated by $\left\{A^{\mathbf{L}}: A \in \mathcal{U}\right\}$, then Corollary 2 in [14] implies

$$
\oint X \mathrm{~d} B e l=\int_{\mathcal{U}} X^{\mathbf{L}} \mathrm{d} \mu \text { and } \oint X \mathrm{~d} P l=\int_{\mathcal{U}} X^{\mathbf{U}} \mathrm{d} \mu .
$$

For $X \in \mathcal{L}(\Omega)$ and $H \in \mathcal{P}(\Omega)^{0}$ the corresponding $H$-cut upper generalized gamble $X^{\mathbf{U}, H} \in$ $\mathcal{L}(\mathcal{U})$ is defined, for every $B \in \mathcal{U}$, as

$$
X^{\mathbf{U}, H}(B)= \begin{cases}\sup _{\omega \in B \cap H} X(\omega), & \text { if } B \cap H \neq \emptyset, \\ 0, & \text { otherwise. }\end{cases}
$$

The following proposition will be useful in the rest of the paper.

Proposition 3.1 Let $\mathcal{U}=\mathcal{P}(\Omega)^{0}$ and $\mu$ be the Möbius inverse on $\mathfrak{A}$ of the belief function Bel, the latter defined on $\mathcal{P}(\Omega)$ and with dual Pl. Then, for every $X \in \mathcal{L}(\Omega)$ and every $H \in \mathcal{P}(\Omega)^{0}$, it holds that

$$
\oint X \mathrm{~d} P l(\cdot \cap H)=\int_{\mathcal{U}} X^{\mathbf{U}, H} \mathrm{~d} \mu .
$$


Proof Since $X \in \mathcal{L}(\Omega)$, there exists a sequence $\left\{X_{n}\right\}_{n \in \mathbb{N}}$ of simple functions in $\mathcal{L}(\Omega)$ converging uniformly to $X$.

We first show that the statement holds for every $X_{n}$. Suppose $X_{n}=\sum_{i=1}^{m} x_{i} \mathbf{1}_{A_{i}}$, where $\left\{A_{1}, \ldots, A_{m}\right\}$ is a partition of $\Omega$ and $x_{1}>\cdots>x_{m}$. Let $I=\left\{i_{1}, \ldots, i_{t}\right\}$ be the subset of indices such that $A_{i_{j}} \cap H \neq \emptyset$ and suppose $x_{i_{1}}>\cdots>x_{i_{t}}$. Then, $X_{n}^{\mathbf{U}, H}$ is a simple function in $\mathcal{L}(\mathcal{U})$ that can be expressed as $X_{n}^{\mathbf{U}, H}=\sum_{j=1}^{t} x_{i_{j}} \mathbf{1}_{C_{j}}$, where $C_{1}=\{B \in \mathcal{U}$ : $\left.B \cap A_{i_{1}} \cap H \neq \emptyset\right\}, C_{j}=\left\{B \in \mathcal{U}: B \cap A_{i_{j}} \cap H \neq \emptyset\right\} \backslash \bigcup_{k=1}^{j-1} C_{k}$, for $j=2, \ldots, t$, and further define $C_{t+1}=\left(\bigcup_{j=1}^{t} C_{j}\right)^{c}$. It is easily seen that $\left\{C_{1}, \ldots, C_{t}, C_{t+1}\right\}$ is a partition of $\mathcal{U}$ contained in $\mathfrak{A}$, possibly discarding $C_{t+1}$ in case it is equal to $\emptyset$.

Define $E_{i}=\bigcup_{k=1}^{i} A_{k}$, for $i=1, \ldots, m$, with $E_{0}=\emptyset$. By the definition of the Choquet integral for simple functions $[8,12]$, we have that

$$
\begin{aligned}
\oint X_{n} \mathrm{~d} P l(\cdot \cap H) & =\sum_{i=1}^{n} x_{i}\left(P l\left(E_{i} \cap H\right)-P l\left(E_{i-1} \cap H\right)\right) \\
& =\sum_{j=1}^{t} x_{i_{j}} \mu\left(C_{j}\right)=\int_{\mathcal{U}} X_{n}^{\mathbf{U}, H} \mathrm{~d} \mu,
\end{aligned}
$$

where the second equality follows since $P l\left(E_{i} \cap H\right)-P l\left(E_{i-1} \cap H\right)$ is equal to $\mu\left(\left(E_{i} \cap\right.\right.$ $\left.H)^{\mathbf{U}} \backslash\left(E_{i-1} \cap H\right)^{\mathbf{U}}\right)$. Moreover, $\left(E_{i} \cap H\right)^{\mathbf{U}} \backslash\left(E_{i-1} \cap H\right)^{\mathbf{U}}$ is either equal to $\emptyset$ or reduces to one of the $C_{j}$, and, for all $B \in C_{j}$, it holds $X_{n}^{\mathbf{U}, H}(B)=x_{i_{j}}=x_{i}$.

Now, since $\left\{X_{n}\right\}_{n \in \mathbb{N}}$ converges to $X$ uniformly, for every $\epsilon>0$, there exists $n_{\frac{\epsilon}{2}} \in \mathbb{N}$ such that for every $n \geq n \frac{\epsilon}{2}$ it holds that $\sup _{\omega \in \Omega}\left|X_{n}(\omega)-X(\omega)\right|<\frac{\epsilon}{2}$.

For every $n \geq n \frac{\epsilon}{2}$ and every $B \in \mathcal{U}$ such that $B \cap H \neq \emptyset$, by condition $(x)$ of Theorem 4.13 in [19], we have that

$$
\begin{aligned}
\left|\sup _{\omega \in B \cap H} X_{n}(\omega)-\sup _{\omega \in B \cap H} X(\omega)\right| & \leq \sup _{\omega \in B \cap H}\left|X_{n}(\omega)-X(\omega)\right| \\
& \leq \sup _{\omega \in \Omega}\left|X_{n}(\omega)-X(\omega)\right|<\frac{\epsilon}{2} .
\end{aligned}
$$

Therefore, since $X_{n}^{\mathbf{U}, H}(B)=X^{\mathbf{U}, H}(B)=0$ for every $B \cap H=\emptyset$, for every $n \geq n \frac{\epsilon}{2}$, it holds that $\sup _{B \in \mathcal{U}}\left|X_{n}^{\mathbf{U}, H}(B)-X^{\mathbf{U}, H}(B)\right| \leq \frac{\epsilon}{2}<\epsilon$, implying that the sequence $\left\{X_{n}^{\mathbf{U}, H}\right\}_{n \in \mathbb{N}}$ of simple $\mathfrak{A}$-measurable functions converges uniformly to $X^{\mathbf{U}, H}$, that is $X^{\mathbf{U}, H}$ is $\mathfrak{A}$-continuous [1].

Finally, by the continuity of both the Choquet integral and the Stieltjes integral with respect to the topology of uniform convergence [19], and the fact that limits keep equalities, we have that

$$
\oint X \mathrm{~d} P l(\cdot \cap H)=\lim _{n \in \mathbb{N}} \oint X_{n} \mathrm{~d} P l(\cdot \cap H)=\lim _{n \in \mathbb{N}} \int_{\mathcal{U}} X_{n}^{\mathbf{U}, H} \mathrm{~d} \mu=\int_{\mathcal{U}} X^{\mathbf{U}, H} \mathrm{~d} \mu .
$$

Notice that all finitely additive probability measures on $\mathcal{P}(\mathcal{U})$ extending $\mu$ give rise to the same $\mathrm{Bel}$ and $\mathrm{Pl}$ on $\mathcal{P}(\Omega)$ through 1 and satisfy the equation in Proposition 3.1. 


\section{Conditioning and coherence}

Given a gamble $X \in \mathcal{L}(\Omega)$ and an event $H \in \mathcal{P}(\Omega)^{0}$, a conditional gamble is a pair $(X, H)$, usually denoted by $X \mid H$, which consists in regarding $X$ under the hypothesis $H$. In particular, a conditional event $E \mid H \in \mathcal{P}(\Omega) \times \mathcal{P}(\Omega)^{0}$ is identified with the conditional gamble $\mathbf{1}_{E} \mid H$, and an unconditional gamble $X \in \mathcal{L}(\Omega)$ is identified with $X \mid \Omega$.

Let $\operatorname{Pl}(\cdot \mid \cdot)$ be a conditional plausibility function on $\mathcal{P}(\Omega) \times \mathcal{H}$ and $\operatorname{Bel}(\cdot \mid \cdot)$ its dual conditional belief function. Denote by $\mathbf{C}_{B e l}(\cdot \mid \cdot)$ and $\mathbf{C}_{P l}(\cdot \mid \cdot)$ the conditional functionals on $\mathcal{L}(\Omega) \times \mathcal{H}$, determined by the Choquet integral with respect to $\operatorname{Bel}(\cdot \mid \cdot)$ and $\operatorname{Pl}(\cdot \mid \cdot)$, respectively: for every $X \in \mathcal{L}(\Omega)$ and $H \in \mathcal{H}$,

$$
\mathbf{C}_{B e l}(X \mid H)=\oint X \mathrm{~d} \operatorname{Bel}(\cdot \mid H) \text { and } \mathbf{C}_{P l}(X \mid H)=\oint X \mathrm{~d} P l(\cdot \mid H) .
$$

Also in the conditional case, it holds that, for every $k \geq 2$, for every $X_{1}, \ldots, X_{k} \in \mathcal{L}(\Omega)$ and $H \in \mathcal{H}$,

$$
\mathbf{C}_{P l}\left(\bigwedge_{i=1}^{k} X_{i} \mid H\right) \leq \sum_{\emptyset \neq I \subseteq\{1, \ldots, k\}}(-1)^{|I|+1} \mathbf{C}_{P l}\left(\bigvee_{i \in I} X_{i} \mid H\right)
$$

Also in this case, $\mathbf{C}_{B e l}(\cdot \mid \cdot)$ satisfies an analogous property, obtained by inverting the inequality and by switching minima and maxima.

In what follows the conditional functionals $\mathbf{C}_{B e l}(\cdot \mid \cdot)$ and $\mathbf{C}_{P l}(\cdot \mid \cdot)$ are said conditional completely monotone and alternating Choquet expectations.

Notice that, $\mathbf{C}_{B e l}(\cdot \mid \cdot)$ and $\mathbf{C}_{P l}(\cdot \mid \cdot)$ are dual since, for every $X \in \mathcal{L}(\Omega)$ and $H \in \mathcal{H}$, it holds that $\mathbf{C}_{B e l}(X \mid H)=-\mathbf{C}_{P l}(-X \mid H)$, therefore we can limit to deal with $\mathbf{C}_{P l}(\cdot \mid \cdot)$.

We stress that a conditional functional $\mathbf{C}_{P l}(\cdot \mid \cdot)$ essentially relies on the structure of its domain. If we want to weaken the hypotheses on the domain we need to introduce a suitable notion of coherence.

Let $\mathcal{G} \subseteq \mathcal{L}(\Omega) \times \mathcal{P}(\Omega)^{0}$ be an arbitrary subset of conditional bounded gambles. Further, let $\mathcal{H}_{\mathcal{G}}$ be the additive class generated by $\left\{H \in \mathcal{P}(\Omega)^{0}: X \mid H \in \mathcal{G}\right\}$. Here we provide a notion of coherence for an assessment $\Psi: \mathcal{G} \rightarrow \mathbb{R}$, showing its characterization in terms of a form of generalized Dutch book.

Definition 4.1 An assessment $\Psi: \mathcal{G} \rightarrow \mathbb{R}$ is $\mathbf{C P l}$-coherent if there exists a conditional plausibility function $P l: \mathcal{P}(\Omega) \times \mathcal{H}_{\mathcal{G}} \rightarrow[0,1]$ inducing the conditional completely alternating Choquet expectation $\mathbf{C}_{P l}: \mathcal{L}(\Omega) \times \mathcal{H}_{\mathcal{G}} \rightarrow \mathbb{R}$ such that, for every $X \mid H \in \mathcal{G}$, it holds that $\mathbf{C}_{P l}(X \mid H)=\Psi(X \mid H)$.

Definition 4.2 An assessment $\Psi: \mathcal{G} \rightarrow \mathbb{R}$ avoids conditional CPI-Dutch book if, for every $n \in \mathbb{N}$, for every $\mathcal{F}=\left\{X_{1}\left|H_{1}, \ldots, X_{n}\right| H_{n}\right\} \subseteq \mathcal{G}$, and every $\lambda_{1}, \ldots, \lambda_{n} \in \mathbb{R}$, the function $G_{\mathcal{F}}: \mathcal{U} \rightarrow \mathbb{R}$ defined as

$$
G_{\mathcal{F}}=\sum_{i=1}^{n} \lambda_{i}\left(X_{i}^{\mathbf{U}, H_{i}}-\Psi\left(X_{i} \mid H_{i}\right) \mathbf{1}_{H_{i}}^{\mathbf{U}}\right),
$$

is such that $\sup _{B \subseteq H_{0}^{0}} G_{\mathcal{F}}(B) \geq 0$, where $H_{0}^{0}=\bigcup_{i=1}^{n} H_{i}$.

The function $G_{\mathcal{F}}$ can be seen as a random gain under partially resolving uncertainty [13]: we admit bets where the gain can be computed only knowing that an event $B \neq \emptyset$ occurs, even if we are not able to identify the true $\omega \in B$. The gain $G_{\mathcal{F}}$ is asked not to be uniformly negative over $B$ 's contained in $H_{0}^{0}$. 
Theorem 4.1 For an assessment $\Psi: \mathcal{G} \rightarrow \mathbb{R}$ the following statements are equivalent:

(i) $\Psi$ is CPl-coherent;

(ii) $\Psi$ avoids conditional CPl-Dutch book.

Proof We first prove the theorem for a finite $\mathcal{G}=\left\{X_{1}\left|H_{1}, \ldots, X_{n}\right| H_{n}\right\}$, showing that (i) is equivalent to (ii). Let $H_{0}^{0}=\bigcup_{i=1}^{n} H_{i}$ and $\mathcal{H}_{\mathcal{G}}$ be the additive class generated by $\left\{H_{1}, \ldots, H_{n}\right\}$.

Condition (i) is equivalent to the existence of a conditional plausibility function $P l$ : $\mathcal{P}(\Omega) \times \mathcal{H}_{\mathcal{G}} \rightarrow[0,1]$ inducing the conditional completely alternating Choquet expectation $\mathbf{C}_{P l}: \mathcal{L}(\Omega) \times \mathcal{H}_{\mathcal{G}} \rightarrow \mathbb{R}$ extending the assessment $\Psi$. Since the additive class $\mathcal{H}_{\mathcal{G}}$ is finite and has top element $H_{0}^{0}$, every conditional plausibility function $P l(\cdot \mid \cdot)$ is in bijection with a minimal agreeing class of plausibility functions $\left\{P l_{0}, \ldots, P l_{k}\right\}$ on $\mathcal{P}(\Omega)$, where $P l_{\alpha}\left(H_{0}^{\alpha}\right)=1$ and $P l_{\alpha}\left(\left(H_{0}^{\alpha}\right)^{c}\right)=0$. By Theorem A in [11], every such $P l_{\alpha}$ is in bijection with a finitely additive probability measure $\mu_{\alpha}$ defined on $\mathfrak{A}$ that, by the Hahn-Banach theorem, can be extended, generally not in a unique way, to a continuous positive linear functional with unit norm $f_{\alpha} \in \mathcal{L}(\mathcal{U})^{*}$. Notice that the restriction of $f_{\alpha}$ to the indicators of events in $\mathcal{P}(\mathcal{U})$ determines a finitely additive probability measure extending $\mu_{\alpha}$, that we continue to denote by $\mu_{\alpha}$. Taking into account Proposition 3.1, the functional $f_{\alpha}$ satisfies, for all $H_{i} \subseteq H_{0}^{\alpha}, f_{\alpha}\left(X_{i}^{\mathbf{U}, H_{i}}\right)=\Psi\left(X_{i} \mid H_{i}\right) f_{\alpha}\left(\mathbf{1}_{H_{i}}^{\mathrm{U}}\right)$.

Hence, condition (i) is equivalent to the existence of a linearly ordered class $\left\{f_{0}, \ldots, f_{k}\right\}$ of elements of $\mathcal{L}(\mathcal{U})^{*}$ with the above properties. In turn, this is equivalent to the solvability of the sequence of systems $\mathcal{S}_{0}, \ldots, \mathcal{S}_{k}$ defined below.

For $\alpha=0$, we search for $f \in \mathcal{L}(\mathcal{U})^{*}$ solving the system

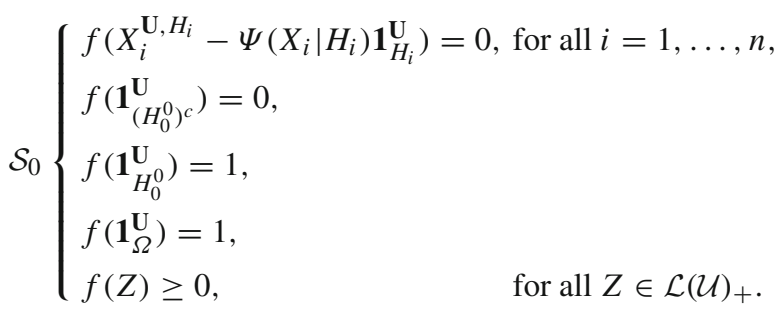

By Theorem 17 in [9], the above system has solution if and only if, for every $\lambda_{1}, \ldots, \lambda_{n}, \lambda_{n+1}, \lambda_{n+2}, \lambda_{n+3} \in \mathbb{R}$, defining the functions $G_{0}, Y_{0}: \mathcal{U} \rightarrow \mathbb{R}$ as

$$
\begin{aligned}
G_{0} & =\sum_{i=1}^{n} \lambda_{i}\left(X_{i}^{\mathbf{U}, H_{i}}-\Psi\left(X_{i} \mid H_{i}\right) \mathbf{1}_{H_{i}}^{\mathbf{U}}\right), \\
Y_{0} & =G_{0}+\lambda_{n+1} \mathbf{1}_{\left(H_{0}^{0}\right)^{c}}^{\mathbf{U}}+\lambda_{n+2} \mathbf{1}_{H_{0}^{0}}^{\mathbf{U}}+\lambda_{n+3} \mathbf{1}_{\Omega}^{\mathbf{U}},
\end{aligned}
$$

it does not happen that

$$
\lambda_{n+2}+\lambda_{n+3}=0 \text { and } Y_{0} \in \operatorname{int}\left(\mathcal{L}(\mathcal{U})_{+}\right),
$$

i.e., we cannot have $\lambda_{n+2}+\lambda_{n+3}=0$ and $\inf _{B \in \mathcal{U}} Y_{0}(B)>0$. Up to a change of sign of $\lambda_{i}$ 's, $\mathcal{S}_{0}$ has solution if and only if it does not happen that $\lambda_{n+2}+\lambda_{n+3}=0$ and $\sup _{B \in \mathcal{U}} Y_{0}(B)<0$. Since

$$
Y_{0}(B)= \begin{cases}G_{0}(B)+\lambda_{n+2}+\lambda_{n+3}, & \text { if } B \subseteq H_{0}^{0}, \\ \lambda_{n+1}+\lambda_{n+3}, & \text { if } B \subseteq\left(H_{0}^{0}\right)^{c}, \\ G_{0}(B)+\lambda_{n+1}+\lambda_{n+2}+\lambda_{n+3}, & \text { otherwise, }\end{cases}
$$


we show that condition 4 does not hold for every $\lambda_{1}, \ldots, \lambda_{n+3} \in \mathbb{R}$ if and only if $\sup _{B \subseteq H_{0}^{0}} G_{0}(B) \geq 0$ for every $\lambda_{1}, \ldots, \lambda_{n} \in \mathbb{R}$.

Suppose $\sup _{B \subseteq H_{0}^{0}} G_{0}(B) \geq 0$ for every $\lambda_{1}, \ldots, \lambda_{n} \in \mathbb{R}$. Then, if $\lambda_{n+2}+\lambda_{n+3}=0$, we have $\sup _{B \subseteq H_{0}^{0}} Y_{0}(B) \geq 0$, and so $\sup _{B \in \mathcal{U}} Y_{0}(B) \geq 0$. To prove the converse, suppose by absurd that for every $\lambda_{1}, \ldots, \lambda_{n+3} \in \mathbb{R}$ with $\lambda_{n+2}+\lambda_{n+3}=0$, one has $\sup _{B \in \mathcal{U}} Y_{0}(B) \geq 0$ and that there exist $\lambda_{1}, \ldots, \lambda_{n}$ such that $\sup _{B \subseteq H_{0}^{0}} G_{0}(B)<0$. Then, keeping $\lambda_{1}, \ldots, \lambda_{n}$ fixed and taking $\lambda_{n+1}<-\sup _{B n H_{0}^{0} \neq \emptyset, B n\left(H_{0}^{0}\right)^{c} \neq \emptyset}\left|G_{0}(B)\right|$ and $-\lambda_{n+2}=\lambda_{n+3}=\lambda_{n+1}$, we get that $\lambda_{n+2}+\lambda_{n+3}=0$ and $\sup _{B \in \mathcal{U}} Y_{0}(B)<0$, contrary to the hypothesis. So, the claim follows.

If $f_{0}$ is a solution of $\mathcal{S}_{0}$, its restriction to indicators of events in $\mathcal{P}(\mathcal{U})$ determines a finitely additive probability measure $\mu_{0}$. In turn, $\mu_{0}$ induces a plausibility function $P l_{0}$ on $\mathcal{P}(\Omega)$ through 1 satisfying $P l_{0}\left(H_{0}^{0}\right)=1, P l_{0}\left(\left(H_{0}^{0}\right)^{c}\right)=0$ and, by Proposition 3.1,

$$
\oint X_{i} \mathrm{~d} P l_{0}\left(\cdot \cap H_{i}\right)=\Psi\left(X_{i} \mid H_{i}\right) P l_{0}\left(H_{i}\right) .
$$

Hence, if $P l_{0}\left(H_{i}\right)>0, \Psi\left(X_{i} \mid H_{i}\right)=\frac{1}{P l_{0}\left(H_{i}\right)} \oint X_{i} \mathrm{~d} P l_{0}\left(\cdot \cap H_{i}\right)=\oint X_{i} \mathrm{~d} P l\left(\cdot \mid H_{i}\right)$.

For $\alpha>0$, let $I_{\alpha}=\left\{i \in\{1, \ldots, n\}: P l_{\beta}\left(H_{i}\right)=0, \beta=0, \ldots, \alpha-1\right\}$ and define $H_{0}^{\alpha}=\bigcup_{i \in I_{\alpha}} H_{i}$. We search for $f \in \mathcal{L}(\mathcal{U})^{*}$ solving the system

$$
\mathcal{S}_{\alpha}:\left\{\begin{array}{l}
f\left(X_{i}^{\mathbf{U}, H_{i}}-\Psi\left(X_{i} \mid H_{i}\right) \mathbf{1}_{H_{i}}^{\mathbf{U}}\right)=0, \text { for all } i \in I_{\alpha}, \\
f\left(\mathbf{1}_{\left(H_{0}^{\alpha}\right)^{c}}^{\mathbf{U}}\right)=0, \\
f\left(\mathbf{1}_{H_{0}^{\alpha}}^{\mathbf{U}}\right)=1, \\
f\left(\mathbf{1}_{\Omega}^{\mathbf{U}}\right)=1, \\
f(Z) \geq 0,
\end{array}\right.
$$

Also in this case, system $\mathcal{S}_{\alpha}$ has solution if and only if for every $\lambda_{i} \in \mathbb{R}, i \in I_{\alpha}$, defining the function $G_{\alpha}: \mathcal{U} \rightarrow \mathbb{R}$ as

$$
G_{\alpha}=\sum_{i \in I_{\alpha}} \lambda_{i}\left(X_{i}^{\mathbf{U}, H_{i}}-\Psi\left(X_{i} \mid H_{i}\right) \mathbf{1}_{H_{i}}^{\mathbf{U}}\right),
$$

we have $\sup _{B \subseteq H_{0}^{\alpha}} G_{\alpha}(B) \geq 0$. As before, from a solution $f_{\alpha}$ we derive the finitely additive probability measure $\mu_{\alpha}$ on $\mathcal{P}(\mathcal{U})$ that induces through 1 the plausibility function $P l_{\alpha}$ on $\mathcal{P}(\Omega)$. Notice that the sequence stops at index $k$ such that $P l_{k}\left(H_{i}\right)>0$ for all $H_{i} \subseteq H_{0}^{k}$.

Finally, since the same $P l(\cdot \mid \cdot)$ determines $\mathbf{C}_{P l}(\cdot \mid \cdot)$ whose restriction to $\mathcal{L}(\Omega) \times \mathcal{H}_{\mathcal{F}}$ extends the restriction $\Psi_{\mid \mathcal{F}}$ for every $\mathcal{F} \subseteq \mathcal{G}$, repeating the previous construction for such a restriction, we have that taking the supremum of all the resulting functions $G_{\alpha}$ for $B \subseteq H_{0}^{\alpha}$ is equivalent to condition (ii).

Now we consider an arbitrary $\mathcal{G}$. We only need to prove (ii) $\Longrightarrow$ (i), as the other implication follows by the proof for the finite case. Indeed, if $\Psi$ is CPl-coherent, then also $\Psi_{\mid \mathcal{F}}$ is, for every finite $\mathcal{F} \subseteq \mathcal{G}$, and this implies (ii).

For every finite $\mathcal{F} \subseteq \mathcal{G}$, let $\mathbb{P}_{\mathcal{F}} \subseteq \prod_{X \mid H \in \mathcal{L}(\Omega) \times \mathcal{H}_{\mathcal{G}}}\left[\inf _{\omega \in \Omega} X(\omega), \sup _{\omega \in \Omega} X(\omega)\right]$, be the set of all inf/sup bounded real-valued functions on $\mathcal{L}(\Omega) \times \mathcal{H}_{\mathcal{G}}$ whose restriction to $\mathcal{L}(\Omega) \times \mathcal{H}_{\mathcal{F}}$ is a conditional completely alternating Choquet expectation extending $\Psi_{\mid \mathcal{F}}$, determined by a conditional plausibility function on $\mathcal{P}(\Omega) \times \mathcal{H}_{\mathcal{F}}$. The set $\mathbb{P}_{\mathcal{F}}$ is not empty by the proof for the finite case and is easily seen to be a closed subset of the compact set $\prod_{X \mid H \in \mathcal{L}(\Omega) \times \mathcal{H}_{\mathcal{G}}}\left[\inf _{\omega \in \Omega} X(\omega), \sup _{\omega \in \Omega} X(\omega)\right]$ endowed with the product topology. Indeed, 
for every net $\left\{\mathbf{D}_{\gamma}\right\}_{\gamma \in \Gamma}$ in $\mathbb{P}_{\mathcal{F}}$ converging pointwise to $\mathbf{D}$, a simple application of properties of limits of real nets and the main Theorem in [16] imply that $\mathbf{D} \in \mathbb{P}_{\mathcal{F}}$.

It is also easily seen that the family $\mathfrak{P}=\left\{\mathbb{P}_{\mathcal{F}}: \mathcal{F}=\left\{X_{1}\left|H_{1}, \ldots, X_{n}\right| H_{n}\right\} \subseteq \mathcal{G}, n \in \mathbb{N}\right\}$, possesses the finite intersection property, thus it holds $\bigcap \mathfrak{P} \neq \emptyset$ and so there exists $\mathbf{C}_{P l} \in$ $\bigcap \mathfrak{P}$ which is a conditional completely alternating Choquet expectation extending $\Psi$.

Acknowledgements Open access funding provided by Università degli Studi di Roma La Sapienza within the 276 CRUI-CAREAgreement. The authors are members of GNAMPA-INdAM. D. Petturiti was partially supported by University of Perugia, Fondo Ricerca di Base 2019, project "Modelli per le decisioni economiche e finanziarie in condizioni di ambiguità ed imprecisione".

Open Access This article is licensed under a Creative Commons Attribution 4.0 International License, which permits use, sharing, adaptation, distribution and reproduction in any medium or format, as long as you give appropriate credit to the original author(s) and the source, provide a link to the Creative Commons licence, and indicate if changes were made. The images or other third party material in this article are included in the article's Creative Commons licence, unless indicated otherwise in a credit line to the material. If material is not included in the article's Creative Commons licence and your intended use is not permitted by statutory regulation or exceeds the permitted use, you will need to obtain permission directly from the copyright holder. To view a copy of this licence, visit http://creativecommons.org/licenses/by/4.0/.

\section{References}

1. Bhaskara Rao, K.P.S., Bhaskara Rao, M.: Theory of Charges. A Study of Finitely Additive Measures. Academic Press, New York (1983)

2. Candeloro, D., Mesiar, R., Sambucini, A.R.: A special class of fuzzy measures: Choquet integral and applications. Fuzzy Sets Syst. 355, 83-99 (2019)

3. Coletti, G., Petturiti, D., Vantaggi, B.: Conditional belief functions as lower envelopes of conditional probabilities in a finite setting. Inf. Sci. 339, 64-84 (2016)

4. Coletti, G., Petturiti, D., Vantaggi, B.: Dutch book rationality conditions for conditional preferences under ambiguity. Ann. Oper. Res. 279(1), 115-150 (2019)

5. Coletti, G., Vantaggi, B.: A view on conditional measures through local representability of binary relations. Int. J. Approx. Reason. 47(1), 268-283 (2008)

6. de Finetti, B.: Theory of Probability 1-2. Wiley, London (1975)

7. Dempster, A.P.: Upper and lower probabilities induced by a multivalued mapping. Ann. Math. Stat. 38(2), 325-339 (1967)

8. Denneberg, D.: Non-Additive Measure and Integral. Kluwer Academic Publisher, Dordrecht (1994)

9. Fan, K.: On system of linear inequalities. In: Kuhn, H.W., Tucker, A.W. (eds.) Linear Inequalities and Related Systems, pp. 99-156. Princeton University Press, Princeton (1956). (AM-38)

10. Gilboa, I., Schmeidler, D.: Additive representations of non-additive measures and the Choquet integral. Ann. Oper. Res. 52(1), 43-65 (1994)

11. Gilboa, I., Schmeidler, D.: Canonical representation of set functions. Math. Oper. Res. 20(1), 197-212 (1995)

12. Grabisch, M.: Set Functions. Games and Capacities in Decision Making. Springer, Berlin (2016)

13. Jaffray, J.Y.: Coherent bets under partially resolving uncertainty and belief functions. Theor. Decis. 26(2), 99-105 (1989)

14. Marinacci, M.: Decomposition and representation of coalitional games. Math. Oper. Res. 21(4), 10001015 (1996)

15. Petturiti, D., Vantaggi, B.: Conditional submodular Choquet expected values and conditional coherent risk measures. Int. J. Approx. Reason. 113, 14-38 (2019)

16. Schmeidler, D.: Integral representation without additivity. Proc. Am. Math. Soc. 97(2), 255-261 (1986)

17. Shafer, G.: A Mathematical Theory of Evidence. Princeton University Press, Princeton (1976)

18. Shafer, G.: Allocations of probability. Ann. Probab. 7(5), 827-839 (1979)

19. Troffaes, M.C.M., de Cooman, G.: Lower Previsions. Wiley Series in Probability and Statistics. Wiley, New York (2014)

Publisher's Note Springer Nature remains neutral with regard to jurisdictional claims in published maps and institutional affiliations. 